\title{
A NEW CONVERGENT ALGORITHM TO APPROXIMATE POTENTIALS FROM FIXED ANGLE SCATTERING DATA*
}

\author{
JUAN A. BARCELÓ ${ }^{\dagger}$, C. CASTRO ${ }^{\dagger}$, T. LUQUE ${ }^{\ddagger}$, AND MARI CRUZ VILELA $\dagger$
}

Abstract. We introduce a new iterative method to recover a real compact supported potential of the Schödinger operator from their fixed angle scattering data. The method combines a fixed point argument with a suitable approximation of the resolvent of the Schödinger operator by partial sums associated to its Born series. The main interest is that, unlike other iterative methods in the literature, each iteration is explicit (and therefore faster computationally) and a rigorous analytical result on the convergence of the iterations is proved. This result requires potentials with small norm in certain Sobolev spaces. As an application we show some numerical experiments that illustrate this convergence.

Key words. Inverse problem, Helmholtz equation, scattering.

AMS subject classifications. 35P25, 35R30, 35J05

1. Introduction and statement of results. We consider the scattering problem for the Schrödinger operator $-\Delta+q$ in $\mathbb{R}^{d}, d \geq 2$, where $q$ is a real valued potential with compact support in $B(0, R)$. Here $B(0, R)$ denotes the ball centred at the origin with radius $R>0$.

Associated to a given wave number $k>0$ and an incident direction $\theta \in S^{d-1}$ we consider the incident wave $u_{i}(x)=e^{i k \theta \cdot x}$. Here $S^{d-1}$ denotes the unit sphere in $\mathbb{R}^{d}$. The outgoing scattering solution $u=u(x, \theta, k)$ with wave number $k$ and direction of propagation $\theta$, is the solution of equation

$$
\left(\Delta+k^{2}\right) u(x)=q(x) u(x), \quad x \in \mathbb{R}^{d},
$$

which can be written as $u=u_{i}+u_{s}$ with $u_{s}(x, \theta, k)$ satisfying the outgoing Sommerfeld radiation condition given by

$$
\partial_{r} u_{s}-i k u_{s}=o\left(r^{-(d-1) / 2}\right), \quad r=|x| \longrightarrow \infty
$$

The function $u_{s}(x, \theta, k)$ named the scattered wave, is the perturbation of $u$ due to the potential. It is well known that for appropriate $q, u_{s}$ satisfies the following asymptotic expression as $|x| \rightarrow \infty$,

$$
u_{s}(x, \theta, k)=c_{d} k^{(d-3) / 2} e^{i k|x|}|x|^{-(d-1) / 2} u_{\infty}\left(\theta^{\prime}, \theta, k\right)+o\left(|x|^{-(d-1) / 2}\right),
$$

where $\theta^{\prime}=x /|x|$ is the reflecting angle and

$$
u_{\infty}\left(\theta^{\prime}, \theta, k\right)=\int_{\mathbb{R}^{d}} e^{-i k \theta^{\prime} \cdot y} q(y) u(y, \theta, k) d y .
$$

The function $u_{\infty}$ is called the scattering amplitude or far-field pattern, and it represents the measurements in the inverse scattering problem. For a successful description of direct and inverse scattering problems we refer the reader to Chapter 5 in [6]. We are interested in recovering the potential $q(x)$ from the knowledge of the scattering amplitudes $u_{\infty}\left(\theta^{\prime}, \theta_{0}, k\right)$, for fixed incident direction $\theta_{0}$ and $\left(\theta^{\prime}, k\right) \in S^{d-1} \times(0, \infty)$, with $\theta^{\prime}=x /|x|$. In fact, by fixed incident direction we mean data for both $\theta=\theta_{0}$ and $\theta=-\theta_{0}$, since we are considering wave

\footnotetext{
* Submitted to the editors 19 February 2018.

Funding: The first and the second author were supported by Spanish Grant MTM2014-57769-C3-2-P, the third by Spanish Grant MTM2014-53850-P2, and the fourth by Spanish Grant MTM2014-57769-C3-1-P.

${ }^{\dagger}$ Departamento de Matemática e Informática aplicadas a las Ingenierías Civil y Naval, Universidad Politécnica de Madrid (juanantonio.barcelo@upm.es, carlos.castro@upm.es, maricruz.vilela@upm.es ).

¥Departamento de Análisis Matemático y Matemática Aplicada, Facultad de Matemáticas, Universidad Complutense de Madrid. (t.luque@ucm.es).
} 
numbers $k>0$. This problem is known as the fixed angle inverse scattering problem and appears naturally in quantum physics. In general, the recovery of information about $q$ from scattering amplitudes is known as inverse scattering problem and it has been studied by several authors. Here we mention some of them that we consider relevant.

Early works on the study of inverse scattering problems can be found in the middle of the last century with the results of Gelfand and Levitan [10], Jost and Kohn [17], and Moses [26]. Later on, Prosser generalized the method in [17] to recover the potential $q$, based on the Born series (nonlinear approximations), when the Friederichs norm of $q$ is small enough (see $[32,33,34,35]$ ). However, the procedures employed in these papers are purely formal, and the smallness condition is difficult to characterize.

In the nineties, Eskin and Ralston (see [7, 8, 9]), and also Stefanov (see [41]) studied the problem of uniqueness. At this time, Päivärinta, Somersalo and Serov introduced new techniques for dealing with the problem of singularities using all the scattering data (see [31, 29, 30]). This problem was also studied by Greenleaf and Uhlmann (see [11]).

In 2001, Ruiz used very precise estimates for the resolvent of the Laplacian to prove that for non-smooth potential the main singularities of the potential (in the scale of Sobolev spaces) are contained in the fixed angle Born approximation, which is a linear approximation of the potential that we define below (see [37]).

More recently, Kilgore, Moskow and Schotland studied the convergence and stability of the Born series and its inverse for several inverse scattering problems (see [24, 19, 20]). They also made numerical studies in [25]. From the numerical point of view, Barceló, Castro and Reyes studied the recovery of a potential from scattering data using a fixed point algorithm which is not justified from the theoretical point of view (see $[2])$.

All these results are for real potentials, the more general case of complex potentials was treated in [23] by Mochizuki and in [3] by Barceló, Faraco, Ruiz and Vargas.

The aim of this paper is to propose an iterative method for recovering a potential $q$ from fixed angle scattering data. More precisely, we obtain a new convergent algorithm that combines two approaches, the nonlinear approximation described by Prosser in [32]-[35], and the fixed point algorithm of Barceló, Castro and Reyes in [2]. It is worth mentioning that, even if the numerical approximations based on these approaches work fine, there are no rigorous proofs in the literature supporting any of them. The interest of the new algorithm is twofold. On one hand, it is computationally faster than the known iterative methods since they involve, at each iteration, the solution of an implicit integral equation ([32]-[35], [2] or [28] for the fixed energy stattering data), while the algorithm proposed here is explicit at each iteration. This makes the implementation simpler and provides a faster algorithm, particularly for 3 -d problems. The second advantage is that we are able to prove the analytical convergence of the iterations rigorously.

To state our main result, we first rewrite the inverse scattering problem in an equivalent integral formulation. Let us define $R_{k}$ the outgoing resolvent operator of the Laplace operator given, in terms of the Fourier transform, by

$$
\widehat{R_{k}(f)}(\xi)=\frac{\widehat{f}(\xi)}{-|\xi|^{2}+k^{2}+i 0} .
$$

Then, $u_{s}$ is the solution of the so-called Lippmann-Schwinger integral equation

$$
u_{s}\left(x, \theta_{0}, k\right)=R_{k}\left(q e^{i k \theta_{0} \cdot(\cdot)}\right)(x)+R_{k}\left(q u_{s}\left(\cdot, \theta_{0}, k\right)\right)(x), \quad x \in \mathbb{R}^{d} .
$$

Moreover, from (1.4) we have that

$$
u_{\infty}\left(\theta, \theta_{0}, k\right)=\int_{\mathbb{R}^{d}} e^{-i k\left(\theta-\theta_{0}\right) \cdot y} q(y) d y+\int_{\mathbb{R}^{d}} e^{-i k \theta \cdot y} q(y) u_{s}\left(y, \theta_{0}, k\right) d y .
$$

The problem is then to find an approximation of the potential $q$ knowing that it satisfies (1.6) and (1.7) parametrized by the scattering data $u_{\infty}\left(\theta, \pm \theta_{0}, k\right)$ with $(\theta, k) \in S^{d-1} \times(0, \infty)$. 


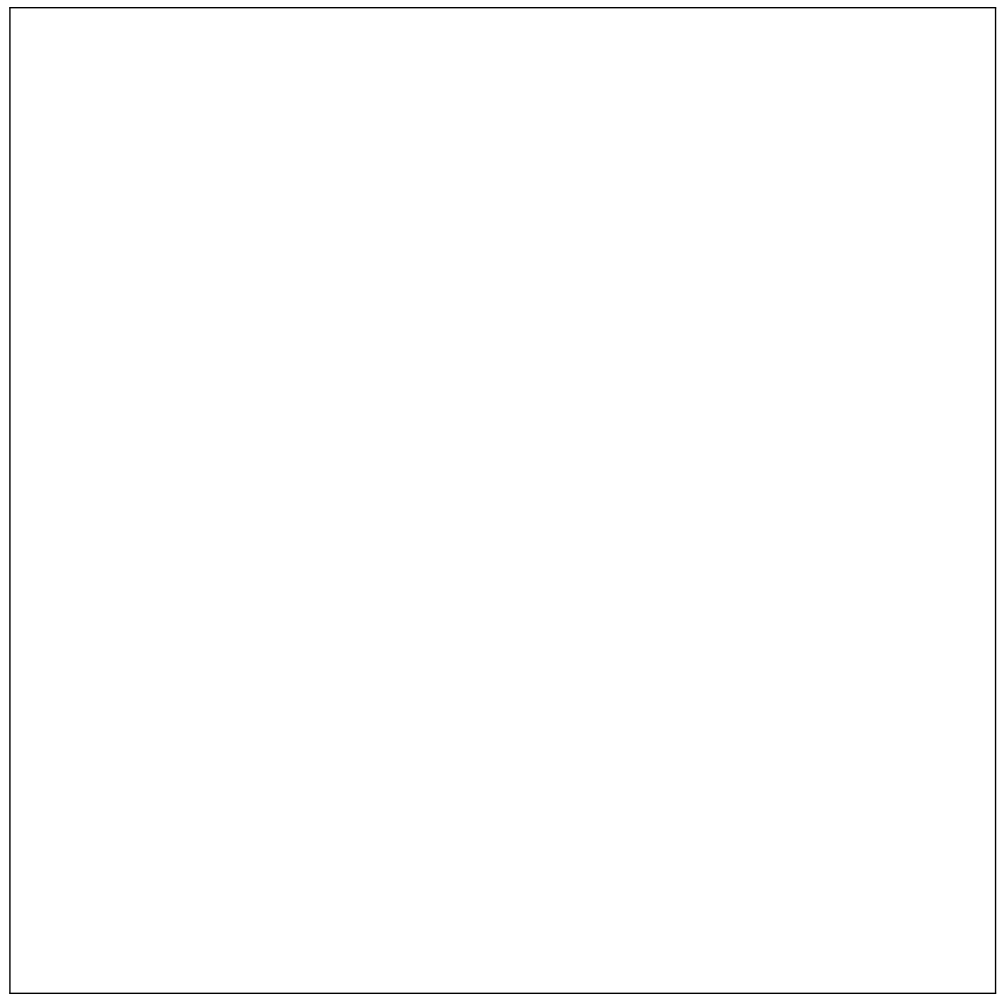

FIG. 1. Ewald spheres are centered at $-k \theta_{0}$ with radius $k$.

If we formally remove the last term in (1.7), the right hand side can be interpreted as a suitable Fourier transform that can be inverted to obtain the so-called Born approximation. More precisely, given $\theta_{0}$ fixed, we have, up to a zero measure set,

$$
\mathbb{R}^{d}=H_{\theta_{0}} \cup H_{-\theta_{0}}=\left\{\xi \in \mathbb{R}^{d}: \xi \cdot \theta_{0}<0\right\} \cup\left\{\xi \in \mathbb{R}^{d}: \xi \cdot \theta_{0}>0\right\} .
$$

Then, for $\xi \in H_{ \pm \theta_{0}}$, there exists unique $\theta(\xi) \in S^{d-1}$ and $k(\xi)>0$ such that

$$
\xi:=k(\xi)\left(\theta(\xi) \mp \theta_{0}\right) \quad(\text { see Figura } 1) .
$$

Let us write

$$
\theta_{0}(\xi)= \begin{cases}\theta_{0}, & \text { if } \xi \in H_{\theta_{0}} \\ -\theta_{0}, & \text { if } \xi \in H_{-\theta_{0}}\end{cases}
$$

Then, the Born approximation for fixed angle scattering data $\theta_{0}$ of a potential $q$ is defined by

$$
\widehat{q_{\theta_{0}}}(\xi)=u_{\infty}\left(\theta(\xi), \theta_{0}(\xi), k(\xi)\right) .
$$

Note that this definition requires scattering data for both $\theta_{0}$ and $-\theta_{0}$.

The algorithm proposed in [2] used this Born approximation to approximate $u_{s}\left(y, \theta_{0}, k\right)$ in (1.7) iteratively. More precisely, a sequence of potentials are defined by $q_{1}=q_{\theta_{0}}$ and

$$
\widehat{q_{n+1}}(\xi)=u_{\infty}\left(\theta(\xi), \theta_{0}(\xi), k(\xi)\right)-\int_{\mathbb{R}^{d}} e^{-i k(\xi) \theta_{0} \cdot y} q_{n}(y) u_{s}^{n}\left(y, \theta_{0}, k(\xi)\right) d y,
$$


where $u_{s}^{n}$ solves

$$
u_{s}^{n}\left(x, \theta_{0}, k\right)=R_{k}\left(q_{n} e^{i k \theta_{0} \cdot(\cdot)}\right)(x)+R_{k}\left(q_{n} u_{s}^{n}\left(\cdot, \theta_{0}, k\right)\right)(x), \quad x \in \mathbb{R}^{d} .
$$

This requires to solve the Lipmann-Schwinger equation (1.6) for all $k(\xi)$ at each iteration. As described in [2] the numerical version of this algorithm converge in few iterations but each one is expensive computationally, even for 2-d problems.

To avoid the solution of equation (1.11) we insert iteratively the Lippmann-Schwinger integral equation (1.6) into (1.4). In this way we obtain

$$
\begin{aligned}
u_{\infty}\left(\theta, \theta_{0}, k\right)= & \int_{\mathbb{R}^{d}} e^{-i k\left(\theta-\theta_{0}\right) \cdot y} q(y) d y \\
& +\sum_{j=1}^{m} \int_{\mathbb{R}^{d}} e^{-i k \theta \cdot y}\left(q R_{k}\right)^{j}\left(q e^{i k \theta_{0} \cdot(\cdot)}\right)(y) d y \\
& +\int_{\mathbb{R}^{d}} e^{-i k \theta \cdot y}\left(q R_{k}\right)^{m}\left(q u_{s}\left(\cdot, \theta_{0}, k\right)\right)(y) d y .
\end{aligned}
$$

Taking $m \rightarrow \infty$, we formally get the Born series

$$
u_{\infty}\left(\theta, \theta_{0}, k\right)=\sum_{j=0}^{\infty} \int_{\mathbb{R}^{d}} e^{-i k \theta \cdot y}\left(q R_{k}\right)^{j}\left(q e^{i k \theta_{0} \cdot(\cdot)}\right)(y) d y .
$$

This is the approach followed by R.T. Prosser. Note that the right hand side of (1.13) is a tensor polynomial where the first term is linear in $q$, the second one bilinear in $q$, the third one trilinear in $q$, and so on. Motivated by this structure, Prosser introduced a classical asymptotic method to recover $q$ based on replacing in (1.13) $u_{\infty}$ by $\varepsilon u_{\infty}$, writing $q$ as the power series $q=\sum_{n=0}^{\infty} \varepsilon^{n} q_{n}$, and then identifying the terms with the same power in $\varepsilon$. This provides a system of equations for $q_{n}$ that can be solved recursively.

Here we follow a different approach. For convenience, we rewrite (1.12) as follows

$$
\widehat{q}(\xi)=\widehat{q_{\theta_{0}}}(\xi)-\sum_{j=1}^{m} \widehat{\mathcal{Q}_{j}(q)}(\xi)-\widehat{q_{m}^{r}}(\xi), \quad \xi \in \mathbb{R}^{d},
$$

where

$$
\widehat{\mathcal{Q}_{j}(q)}(\xi):=\int_{\mathbb{R}^{d}} e^{-i k(\xi) \theta(\xi) \cdot y}\left(q R_{k(\xi)}\right)^{j}\left(q e^{i k(\xi) \theta_{0} \cdot(\cdot)}\right)(y) d y
$$

and

$$
\widehat{q_{m}^{r}}(\xi):=\int_{\mathbb{R}^{d}} e^{-i k(\xi) \theta(\xi) \cdot y}\left(q R_{k(\xi)}\right)^{m}\left(q u_{s}\left(\cdot, \theta_{0}, k\right)\right)(y) d y .
$$

The convergence of this series suggests that the last term in equation (1.14) should be small for large $m$. Based on this idea we consider the following family of reduced equations for $q_{m}$, where we have removed this last term,

$$
\left.\widehat{q_{m}}(\xi)=\widehat{q_{\theta_{0}}}(\xi)-\sum_{j=1}^{m} \widehat{\mathcal{Q}_{j}\left(q_{m}\right.}\right)(\xi), \quad \xi=k(\xi)\left(\theta(\xi) \mp \theta_{0}\right)
$$

with $(\theta, k) \in S^{d-1} \times(0, \infty)$. These reduced equations have the advantage that they do not involve $u_{s}$, avoiding the solution of the Lipmann-Schwinger equation (1.6). However, for each parameter $(\theta, k) \in S^{d-1} \times(0, \infty)$, 
equation (1.17) is still nonlinear in $q_{m}$. Moreover, it is not clear if there exists a unique function $q_{m}$ satisfying (1.17).

Here we propose a fixed point procedure to find approximations of $q_{m}$. More precisely we introduce the linear operator $\mathcal{L}_{m}$ defined by

$$
\widehat{\mathcal{L}_{m}(q)}(\xi):=\widehat{q_{\theta_{0}}}(\xi)-\sum_{j=1}^{m} \widehat{\mathcal{Q}_{j}(q)}(\xi),
$$

Then, if $q_{m}$ is solution of (1.17), it must be also a fixed point of $\mathcal{L}_{m}$ and we can try the usual iterative method based on powers of $\mathcal{L}_{m}$ to approximate $q_{m}$. This requires in particular that $\mathcal{L}_{m}(q)$ is of compact support. Therefore, instead of $\mathcal{L}_{m}$ we consider the modified operator

$$
\mathcal{T}_{m}(q):=\phi \mathcal{L}_{m}(q)=\phi q_{\theta_{0}}-\phi \sum_{j=1}^{m} \mathcal{Q}_{j}(q)
$$

where $\phi \in \mathcal{C}^{\infty}$ is a cut-off function with compact support satisfying

$$
\phi(x)=1 \text {, if }|x|<R \text { and } \phi(x)=0 \text {, if }|x|>2 R .
$$

We are now ready to state the main results in this paper. For each $m \in \mathbb{N}$, we consider the sequence $\left\{q_{m, \ell}\right\}_{\ell \in \mathbb{N}}$ defined recursively by

$$
\left\{\begin{array}{l}
q_{m, 1}=0, \\
q_{m, \ell+1}=\mathcal{T}_{m}\left(q_{m, \ell}\right), \quad \ell \geq 1 .
\end{array}\right.
$$

We note that $q_{m, 2}=\phi q_{\theta_{0}}$, which is a good approximation to a potential $q$ with support in $B(0, R)$.

The purpose of this paper is to prove that the sequence of approximations $\left\{q_{m, \ell}\right\}_{m, \ell \in \mathbb{N}}$ converges to the potential $q$ in some sense. More precisely, we will prove the following theorem.

THEOREM 1.1. For $d \geq 2$ and $\alpha$ satisfying

$$
0<\alpha \leq 1, \quad \text { and } \quad \frac{d}{2}-\frac{d}{d-1}<\alpha<\frac{d}{2}
$$

let $q \in W^{\alpha, 2}\left(\mathbb{R}^{d}\right)$ be a real valued function with compact support in $B(0, R)$ and such that

$$
\|q\|_{W^{\alpha, 2}}<A
$$

for an appropriate constant $A:=A(d, \alpha, R)>0$ small enough (see Remark 1.2). For each $m \in \mathbb{N}$, let $\left\{q_{m, \ell}\right\}_{\ell \in \mathbb{N}}$ be the sequence defined by (1.20). Then, there exists $q_{m} \in W^{\alpha, 2}\left(\mathbb{R}^{d}\right)$ satisfying

$$
q_{m}=\lim _{\ell \rightarrow \infty} q_{m, \ell} \quad \text { in } W^{\alpha, 2}\left(\mathbb{R}^{d}\right) .
$$

Moreover, the sequence $\left\{q_{m}\right\}_{m \in \mathbb{N}}$ satisfies

$$
\lim _{m \rightarrow \infty} q_{m}=q \quad \text { in } W^{\alpha, 2}\left(\mathbb{R}^{d}\right) .
$$

Remark 1.2. Following the proof of Theorem 1.1, one can see that the smallness condition given in (1.22) is

$$
\|q\|_{W^{\alpha, 2}}<A=\min \left(\frac{1}{C_{1}}, \frac{1}{C_{2}}, \frac{1}{2 C_{1} C_{3} C_{4}}, \frac{1}{2^{2+\frac{d-1}{2}\left(\frac{1}{2}-\frac{\alpha}{d}\right)} C_{5}}\right),
$$

where $C_{1}, C_{2}, C_{3}, C_{4}$ and $C_{5}$ are the constants that appear in (2.4), (2.7), (2.8), (2.9) and (2.22) respectively (see below).

Remark 1.3. Conditions in (1.21) imply that $d<5$, and therefore Theorem 1.1 is valid for $2 \leq d \leq 4$. Moreover, for $d=2$, the result holds if $0<\alpha<1$, for $d=3$, if $0<\alpha \leq 1$, and for $d=4$, if $2 / 3<\alpha \leq 1$.

Remark 1.4. In the proof of Theorem 1.1 (see (2.19) below) we will see that the convergence of the sequence $\left\{q_{m}\right\}_{m \in \mathbb{N}}$ to potential $q$ is faster than $\left(C_{2}\|q\|_{W^{\alpha, 2}}\right)^{m}$, where $C_{2}$ is the constant that appears in (2.7) (see below). 
Notation. For $\alpha \in \mathbb{R}$ we introduce the fractional differentiation operator

$$
\Lambda^{\alpha}=(1-\Delta)^{\alpha / 2}=\mathcal{F}^{-1}\langle\xi\rangle^{\alpha} \mathcal{F},
$$

where $\mathcal{F}$ denotes the Fourier transform and $\langle\xi\rangle=\left(1+|\xi|^{2}\right)^{1 / 2}$.

We use the Sobolev spaces

$$
W^{\alpha, p}\left(\mathbb{R}^{d}\right)=\left\{f \in \mathcal{S}^{\prime}\left(\mathbb{R}^{d}\right): \Lambda^{\alpha} f \in L^{p}\left(\mathbb{R}^{d}\right)\right\}, \quad \alpha \in \mathbb{R}, 1 \leq p \leq \infty,
$$

and also their weighted versions

$$
W_{\delta}^{\alpha, p}\left(\mathbb{R}^{d}\right)=\left\{f \in \mathcal{S}^{\prime}\left(\mathbb{R}^{d}\right): \Lambda^{\alpha} f \in L_{\delta}^{p}\left(\mathbb{R}^{d}\right)\right\}, \quad \alpha, \delta \in \mathbb{R}, 1 \leq p \leq \infty,
$$

where $L_{\delta}^{p}\left(\mathbb{R}^{d}\right)=\left\{f:\langle x\rangle^{\delta} f \in L^{p}\left(\mathbb{R}^{d}\right)\right\}$.

Throughout this paper $C$ will denote a positive constant that may change from line to line and depend on some parameters such as $d, \alpha$ or $R$. This dependence will be indicated when relevant. In order to know in detail the required smallness condition on the potential $q$, in some cases, we will name constants by $C_{n}$ with $n$ varying in $\mathbb{N}$. Moreover we will write $C_{n_{1}, \ldots, n_{m}}$ to indicate the product $C_{n_{1}} \ldots C_{n_{m}}, n_{1}, \ldots n_{m} \in \mathbb{N}$.

The rest of this paper is organized as follows. The proof of Theorem 1.1 is given in section 2, and also several lemmas needed in the proof. In section 3 we illustrate these results with several numerical experiments.

2. Proofs. This section is devoted to the proof of Theorem 1.1. We split it into two subsections. The first one contains the proof itself and the statement of two results (see Propositions 2.1 and 2.2 below) which are the key points in the proof. The proofs of these propositions are quite technical, and require of several known results, so we postpone them to the second subsection.

2.1. Proof of Theorem 1.1. The first key point in the proof of Theorem 1.1 are some estimates for a family of operators that generalize the operators $\mathcal{Q}_{j}$ given in (1.15). follows

For every $j \in \mathbb{N}$, we introduce the following multilinear operator defined via its Fourier transform as

$$
\mathcal{P}_{j}(\mathbf{f})(x)=\int_{\mathbb{R}^{d}} e^{i x \cdot \xi} \widehat{\mathcal{P}_{j}(\mathbf{f})}(\xi) d \xi=\left(\int_{H_{\theta_{0}}}+\int_{H_{-\theta_{0}}}\right) e^{i x \cdot \xi \widehat{\mathcal{P}_{j}(\mathbf{f})}(\xi)} d \xi,
$$

where $\mathbf{f}=\left(f_{1}, f_{2}, \ldots, f_{j+1}\right), \theta_{0} \in S^{d-1}$ and $H_{ \pm \theta_{0}}$ is given in (1.8).

Taking into account that any $\xi \in H_{ \pm \theta_{0}}$ can be written in a unique way as $\xi=k\left(\theta \mp \theta_{0}\right)$, with $k=k(\xi)>0$ and $\theta=\theta(\xi) \in S^{d-1}$, we define

$$
\widehat{\mathcal{P}_{j}(\mathbf{f})}(\xi):=\int_{\mathbb{R}^{d}} e^{-i k \theta \cdot y}\left(f_{j+1} R_{k} \ldots f_{2} R_{k}\right)\left(f_{1} e^{i k \theta_{0} \cdot(\cdot)}\right)(y) d y,
$$

Observe that in the particular case $f_{i}=q, i=1,2, \cdots, j+1$, we have that

$$
\widehat{\mathcal{P}_{j}(\mathbf{f})}(\xi)=\widehat{\mathcal{Q}_{j}(q)}(\xi), \quad \xi \in \mathbb{R}^{d} .
$$

Proposition 2.1. Let $d \geq 2$ and $\alpha$ satisfying (1.21). For each $j \in \mathbb{N}$ fixed, let $\mathbf{f}=\left(f_{1}, f_{2}, \cdots f_{j+1}\right)$ with $f_{\ell} \in W^{\alpha, 2}\left(\mathbb{R}^{d}\right)$ and compactly supported with support in $B(0, R)$, for $\ell=1, \ldots j+1$. Then, there exits a constant $C_{1}:=C_{1}(d, \alpha, R)$ such that

$$
\left\|\mathcal{P}_{j}(\mathbf{f})\right\|_{W^{\alpha, 2}} \leq C_{1}^{j} \prod_{\ell=1}^{j+1}\left\|f_{\ell}\right\|_{W^{\alpha, 2}} .
$$


The other key point in the proof of Theorem 1.1 concerns with the error term $q_{m}^{r}$ defined in (1.16).

Proposition 2.2. For $d \geq 2$ and $\alpha$ satisfying

$$
0<\alpha \leq 1, \quad \text { and } \quad \frac{d}{2}-2<\alpha<\frac{d}{2},
$$

let $q \in W^{\alpha, 2}\left(\mathbb{R}^{d}\right)$ be a real valued function with compact support in $B(0, R)$ and such that

$$
\|q\|_{W^{\alpha, 2}}<\frac{1}{2^{2+\frac{d-1}{2}\left(\frac{1}{2}-\frac{\alpha}{d}\right)} C_{5}}
$$

where $C_{5}$ is the constant that appears in (2.22). Then, for every $m \in \mathbb{N}$ there exists a constant $C_{2}:=$ $C_{2}(d, \alpha, R)$ such that

$$
\left\|q_{m}^{r}\right\|_{W^{\alpha, 2}} \leq C_{2}^{m}\|q\|_{W^{\alpha, 2}}^{m+1} .
$$

We also need to control the Born approximation defined in (1.10).

Corollary 2.3. For $d \geq 2$ and $\alpha$ satisfying (1.21), let $q \in W^{\alpha, 2}\left(\mathbb{R}^{d}\right)$ be a real valued function with compact support in $B(0, R)$ satisfying (2.6). Then, there exists a constant $C_{3}:=C_{3}(d, \alpha, R)$ such that

$$
\left\|q_{\theta_{0}}\right\|_{W^{\alpha, 2}}<C_{3}\|q\|_{W^{\alpha, 2}} .
$$

Proof. From (1.14) with $m=1$, we have that

$$
\left\|q_{\theta_{0}}\right\|_{W^{\alpha, 2}} \leq\|q\|_{W^{\alpha, 2}}+\left\|\mathcal{Q}_{1}(q)\right\|_{W^{\alpha, 2}}+\left\|q_{1}^{r}\right\|_{W^{\alpha, 2}} .
$$

The result follows from here using (2.3) and (2.4) for $j=1,(2.7)$ for $m=1$, and (2.6).

Finally, we need the following result concerning the product of functions in Sobolev spaces due to Zolesio (see [44]).

Lemma 2.4. ([12, Theorem 1.4.4.2]) Let $0 \leq \alpha \leq s$ and $s>d / 2$, and let $\phi$ be the cut-off function defined in (1.19), then there exists a constant $C_{4}:=C_{4}(d, \alpha, R)>0$ such that

$$
\|\phi g\|_{W^{\alpha, 2}} \leq C\|\phi\|_{W^{s, 2}}\|g\|_{W^{\alpha, 2}} \leq C_{4}\|g\|_{W^{\alpha, 2}}, \quad \forall g \in W^{\alpha, 2}\left(\mathbb{R}^{d}\right) .
$$

Proof of Theorem 1.1. We split the proof in three steps. In the first one we will prove that for each $m \in \mathbb{N}$, the sequence $\left\{q_{m, \ell}\right\}_{\ell \in \mathbb{N}}$ is bounded in the space $W^{\alpha, 2}\left(\mathbb{R}^{d}\right)$. In the second one we will see that such sequence is a Cauchy sequence in that space, thus it converges to a function $q_{m}$. Finally, in the third one we will prove that $q$ is the limit in $W^{\alpha, 2}\left(\mathbb{R}^{d}\right)$ of $q_{m}$ as $m$ goes to infinity.

STEP 1. We will prove the boundedness by induction on $\ell$. Moreover, for each $m \in \mathbb{N}$, we will prove that

$$
\left\|q_{m, \ell}\right\|_{W^{\alpha, 2}} \leq 2 C_{3,4}\|q\|_{W^{\alpha, 2}}, \quad \forall \ell \geq 2,
$$

whenever $\alpha$ satisfies $(1.21)$ and $q \in W^{\alpha, 2}\left(\mathbb{R}^{d}\right)$ is a real valued function with compact support in $B(0, R)$ satisfying (2.6) and such that

$$
\|q\|_{W^{\alpha, 2}} \leq \frac{1}{2 C_{1,3,4}\left(1+2 C_{4}\right)} .
$$

Here $C_{3,4}=C_{3} C_{4}$ with $C_{3}$ and $C_{4}$ being the constants that appear in (2.8) and (2.9) respectively. Similarly it is defined $C_{1,3,4}$. 
From (1.20) and (1.18), we have that

$$
\left\|q_{m, 2}\right\|_{W^{\alpha, 2}}=\left\|\phi q_{\theta_{0}}\right\|_{W^{\alpha, 2}} .
$$

Using (2.9) and (2.8) we get

$$
\left\|q_{m, 2}\right\|_{W^{\alpha, 2}} \leq C_{3,4}\|q\|_{W^{\alpha, 2}} \leq 2 C_{3,4}\|q\|_{W^{\alpha, 2}},
$$

for $\alpha$ and $q$ under the assumptions of Corollary 2.3.

Arguing as before, from (1.20), (1.18), (2.9) and (2.8) we also get

$$
\begin{aligned}
\left\|q_{m, \ell+1}\right\|_{W^{\alpha, 2}} & \leq\left\|\phi q_{\theta_{0}}\right\|_{W^{\alpha, 2}}+\left\|\phi \sum_{j=1}^{m} \mathcal{Q}_{j}\left(q_{m, \ell}\right)\right\|_{W^{\alpha, 2}} \\
& \leq C_{3,4}\|q\|_{W^{\alpha, 2}}+C_{4} \sum_{j=1}^{m}\left\|\mathcal{Q}_{j}\left(q_{m, \ell}\right)\right\|_{W^{\alpha, 2}} .
\end{aligned}
$$

From here, using identity (2.3), (2.4) and the induction hypothesis (2.10), we obtain

$$
\begin{aligned}
\left\|q_{m, \ell+1}\right\|_{W^{\alpha, 2}} & \leq C_{3,4}\|q\|_{W^{\alpha, 2}}+C_{4} \sum_{j=1}^{m} C_{1}^{j}\left\|q_{m, \ell}\right\|_{W^{\alpha, 2}}^{j+1} \\
& \leq C_{3,4}\|q\|_{W^{\alpha, 2}}+C_{4} \sum_{j=1}^{m} C_{1}^{j}\left(2 C_{3,4}\right)^{j+1}\|q\|_{W^{\alpha, 2}}^{j+1} .
\end{aligned}
$$

Estimate (2.10) follows from here since for $q$ satisfying (2.11) we have that

$$
2 C_{4} \sum_{j=1}^{m}\left(2 C_{1,3,4}\|q\|_{W^{\alpha, 2}}\right)^{j} \leq 1 .
$$

STEP 2. From (1.20) and (1.18), using (2.9), we have that

$$
\left\|q_{m, \ell+1}-q_{m, n+1}\right\|_{W^{\alpha, 2}} \leq C_{4}\left\|\sum_{j=1}^{m}\left(\mathcal{Q}_{j}\left(q_{m, \ell}\right)-\mathcal{Q}_{j}\left(q_{m, n}\right)\right)\right\|_{W^{\alpha, 2}} .
$$

From here, using (2.3), the fact that $\mathcal{P}_{j}$ is a multilinear operator and the triangular inequality, we get

$$
\left\|q_{m, \ell+1}-q_{m, n+1}\right\|_{W^{\alpha, 2}} \leq C_{4} \sum_{j=1}^{m} \sum_{i=1}^{j+1}\left\|\mathcal{P}_{j}\left(\mathbf{f}_{m, \ell, n, i}\right)\right\|_{W^{\alpha, 2}},
$$

where

$$
\mathbf{f}_{m, \ell, n, i}=(q_{m, \ell}, \ldots q_{m, \ell}, \underbrace{q_{m, \ell}-q_{m, n}}_{i-\text { position }}, q_{m, n}, \ldots, q_{m, n}) .
$$

Using (2.4) and (2.10) in (2.12) we obtain

$$
\begin{aligned}
\left\|q_{m, \ell+1}-q_{m, n+1}\right\|_{W^{\alpha, 2}} & \leq C_{4} \sum_{j=1}^{m}(j+1)\left(2 C_{1,3,4}\|q\|_{W^{\alpha, 2}}\right)^{j}\left\|q_{m, \ell}-q_{m, n}\right\|_{W^{\alpha, 2}} \\
& \leq B\left\|q_{m, \ell}-q_{m, n}\right\|_{W^{\alpha, 2}},
\end{aligned}
$$


with

$$
B=\frac{C_{4}}{\left(1-2 C_{1,3,4}\|q\|_{W^{\alpha, 2}}\right)^{2}} .
$$

Observe that $B<1$ whenever $\alpha$ satisfies (1.21) and $q \in W^{\alpha, 2}\left(\mathbb{R}^{d}\right)$ is a real valued function with compact support in $B(0, R)$ satisfying (2.6), (2.11) and

$$
\|q\|_{W^{\alpha, 2}}<\frac{1+\sqrt{C_{4}}}{2 C_{1,3,4}} .
$$

Therefore, for such a $q$, we have that $\left\{q_{m, \ell}\right\}_{\ell \in \mathbb{N}}$ is a Cauchy sequence. As a consequence, there exits $q_{m} \in W^{\alpha, 2}\left(\mathbb{R}^{d}\right)$ such that

$$
q_{m}=\lim _{\ell \rightarrow \infty} q_{m, \ell}, \quad \text { in } W^{\alpha, 2}\left(\mathbb{R}^{d}\right) .
$$

From (2.10), we have that $q_{m}$ satisfies

$$
\left\|q_{m}\right\|_{W^{\alpha, 2}} \leq 2 C_{3,4}\|q\|_{W^{\alpha, 2}} .
$$

Moreover,

$$
q_{m}=\phi q_{\theta_{0}}-\phi \sum_{j=1}^{m} \mathcal{Q}_{j}\left(q_{m}\right),
$$

since arguing as we did to get (2.13), we obtain

$$
\left\|q_{m, \ell+1}-\left(\phi q_{\theta_{0}}-\phi \sum_{j=1}^{m} \mathcal{Q}_{j}\left(q_{m}\right)\right)\right\|_{W^{\alpha, 2}}<\left\|q_{m, \ell}-q_{m}\right\|_{W^{\alpha, 2}} .
$$

STEP 3. For $\alpha$ satisfying (1.21) and $q \in W^{\alpha, 2}\left(\mathbb{R}^{d}\right)$ being a real valued function with compact support in $B(0, R)$ satisfying (2.6), (2.11) and (2.14), from (2.16), (1.14) and (1.19), since $\operatorname{supp}(q) \subset B(0, R)$, we have that

$$
\left\|q_{m}-q\right\|_{W^{\alpha, 2}} \leq\left\|\phi \sum_{j=1}^{m}\left(\mathcal{Q}_{j}\left(q_{m}\right)-\mathcal{Q}_{j}(q)\right)\right\|_{W^{\alpha, 2}}+\left\|\phi q_{m}^{r}\right\|_{W^{\alpha, 2} .}
$$

From here, arguing as we did to get (2.13), we obtain

$$
\left\|q_{m}-q\right\|_{W^{\alpha, 2}} \leq D\left\|q_{m}-q\right\|_{W^{\alpha, 2}}+\left\|\phi q_{m}^{r}\right\|_{W^{\alpha, 2}},
$$

where

$$
D=C_{4} \sum_{j=1}^{m} C_{1}^{j} \sum_{i=1}^{j+1}\left\|q_{m}\right\|_{W^{\alpha, 2}}^{i-1}\|q\|_{W^{\alpha, 2}}^{j+1-i} .
$$

Using (2.15), we have that

$$
D \leq C_{4} \sum_{j=1}^{m}\left(C_{1}\|q\|_{W^{\alpha, 2}}\right)^{j} \sum_{i=1}^{j+1}\left(2 C_{3,4}\right)^{i-1}
$$

and therefore

$$
D \leq \begin{cases}C_{4} \sum_{j=1}^{m}(j+1)\left(C_{1}\|q\|_{W^{\alpha, 2}}\right)^{j}, & \text { if } 2 C_{1,3,4} \leq 1 \\ C_{4} \sum_{j=1}^{m}(j+1)\left(2 C_{1,3,4}\|q\|_{W^{\alpha, 2}}\right)^{j}, & \text { if } 2 C_{1,3,4} \geq 1\end{cases}
$$




$$
\|q\|_{W^{\alpha, 2}}<\min \left(\frac{1}{C_{1}}, \frac{1}{2 C_{1,3,4}}\right) .
$$

Using this in (2.17) we get

$$
\left\|q_{m}-q\right\|_{W^{\alpha, 2}} \leq 2\left\|\phi q_{m}^{r}\right\|_{W^{\alpha, 2} .}
$$

Finally, using (2.9) and (2.7) we have that

$$
\left\|q_{m}-q\right\|_{W^{\alpha, 2}} \leq 2 C_{4} C_{2}^{m}\|q\|_{W^{\alpha, 2}}^{m+1} .
$$

The result follows from here if $q$ satisfies (2.6), (2.11), (2.14), (2.18), and also $\|q\|_{W^{\alpha, 2}}<1 / C_{2}$.

2.2. Proofs of the key points. In this subsection we give the proofs of Propositions 2.1 and 2.2. They require of several known estimates for different operators in weighted Sobolev spaces.

We will need to know the behaviour of the outgoing resolvent of the laplacian denoted by $R_{k}$ (see (1.5)) for $k$ small given in the following lemma.

Lemma 2.5. ([22, Chapter VI], [21, lemma 21.4].). Let $\alpha \in[0,2], k \in[0, b], b>0$ and $\delta>1$. Then

$$
\left\|R_{k} f\right\|_{W_{-\delta}^{\alpha, 2}} \leq C_{b, \delta}\|f\|_{L_{\delta}^{2}} .
$$

For $\theta_{0} \in S^{d-1}$ and $k>0$ fixed, we introduce the following operator involving the outgoing resolvent of the laplacian,

$$
R_{k, \theta_{0}} f(x)=e^{-i k \theta_{0} \cdot x} R_{k}\left(f(\cdot) e^{i k \theta_{0} \cdot(\cdot)}\right)(x) .
$$

The following result can be obtained by interpolation of several estimates due to Agmon (see [1]), Kenig, Ruiz and Sogge (see [18]), Ruiz and Vega (see[39]), and Barceló, Ruiz and Vega (see [4]). For details we refer the reader to [37].

Lemma 2.6. ([37, Lemma 3.4]). Let $\alpha \geq 0, r$ and $t$ such that $0 \leq \frac{1}{t}-\frac{1}{2} \leq \frac{1}{d+1}$ and $0 \leq \frac{1}{2}-\frac{1}{r} \leq \frac{1}{d+1}$, then there exist $\delta>1$ and a constant $C_{5}:=C_{5}(\delta)$ such that

$$
\left\|R_{k, \theta_{0}} f\right\|_{W_{-\delta}^{\alpha, r}} \leq C_{5} k^{-1+\frac{d-1}{2}\left(\frac{1}{t}-\frac{1}{r}\right)}\|f\|_{W_{\delta}^{\alpha, t}} .
$$

We also introduce the restriction operator given by

$$
S_{k, \theta_{0}} f(\theta)=\int_{\mathbb{R}^{d}} e^{-i k\left(\theta-\theta_{0}\right) \cdot y} f(y) d y, \quad \theta \in S^{d-1} .
$$

The following lemma is a consequence of Theorem 3(c) in [4].

Lemma 2.7. If $\delta>1 / 2$ then, there exists $C>0$ such that

$$
\left\|S_{k, \theta_{0}} f\right\|_{L^{2}\left(S^{d-1}\right)} \leq C k^{-\frac{d-1}{2}}\|f\|_{L_{\delta}^{2}} .
$$

Using the previous lemma and the Stein-Tomas restriction theorem(see [42]) we can get the following result which generalizes the previous one.

Lemma 2.8. ([37, Lemma 3.7]). Let $\alpha \geq 0$ and $t$ satisfying $0 \leq \frac{1}{t}-\frac{1}{2} \leq \frac{1}{d+1}$; then there exists $\delta(t)>0$ and $C:=C_{t}>0$ such that for $w(\theta)=\left|\theta-\theta_{0}\right|^{\alpha}$, we have

$$
\left\|w S_{k, \theta_{0}} f\right\|_{L^{2}\left(S^{d-1}\right)} \leq C k^{\frac{d-1}{2}\left(\frac{1}{t}-\frac{3}{2}\right)-\alpha}\|f\|_{W_{\delta(t)}^{\alpha, t}}
$$


Now we state the following result concerning the product of functions in weighted Sobolev spaces due to Zolesio (see [44]). For a more general version and details we refer the reader to Theorem 1.4.4.2 (pp. 28) of [12]. See also Proposition D.3 (pp. 182) of [36] for functions compactly supported.

LEMma 2.9. Let $f$ be compactly supported and $\delta \in \mathbb{R}$.

(i) For a satisfying

$$
0 \leq \alpha \quad \text { and } \quad \alpha>\frac{d}{2}-2
$$

or

$$
0<\alpha \quad \text { and } \quad \alpha \geq \frac{d}{2}-2,
$$

we have that there exists a constant $C:=C(\operatorname{supp}(f))$ such that

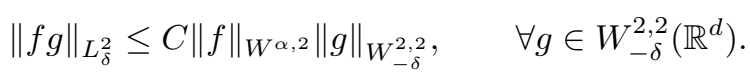

(ii) For $\alpha, t$ and $r$ satisfying

$$
1 \leq t<\min (2, r) \quad \text { and } \quad 0 \leq \frac{1}{2}+\frac{1}{r}-\frac{1}{t} \leq \frac{\alpha}{d},
$$

or

$$
1 \leq t \leq \min (2, r) \quad \text { and } \quad 0 \leq \frac{1}{2}+\frac{1}{r}-\frac{1}{t}<\frac{\alpha}{d},
$$

we have that there exists a constant $C:=C(\operatorname{supp}(f))$ such that

$$
\|f g\|_{W_{\delta}^{\alpha, t}} \leq C\|f\|_{W^{\alpha, 2}}\|g\|_{W_{-\delta}^{\alpha, r},} \quad \forall g \in W_{-\delta}^{\alpha, r}\left(\mathbb{R}^{d}\right) .
$$

(iii) For $\alpha, \beta$ and $p$ satisfying

$$
0 \leq \beta \leq \min (\alpha, 2), \quad 1 \leq p<2 \quad \text { and } \quad 0 \leq d\left(1-\frac{1}{p}\right) \leq \alpha+2-\beta,
$$

we have that there exists a constant $C:=C(\operatorname{supp}(f))$ such that

$$
\|f g\|_{W^{\beta, p}} \leq C\|f\|_{W^{\alpha, 2}}\|g\|_{W_{-\delta}^{2,2}}, \quad \forall g \in W_{-\delta}^{2,2}\left(\mathbb{R}^{d}\right) .
$$

Proof of Proposition 2.1. From (1.8), for a fixed $\theta_{0} \in S^{d-1}$ we have that

$$
\left\|\mathcal{P}_{j}(\mathbf{f})\right\|_{W^{\alpha, 2}}^{2}=\left(\int_{H_{\theta_{0}}}+\int_{H_{-\theta_{0}}}\right)\langle\xi\rangle^{2 \alpha}\left|\widehat{\mathcal{P}_{j}(\mathbf{f})}(\xi)\right|^{2} d \xi=I+I I .
$$

We will prove the estimate for term $I$, the other one is obtained in a similar way.

Observe that in $H_{\theta_{0}}$ we can make the following change of variables

$$
\xi=k\left(\theta-\theta_{0}\right), \quad d \xi=k^{d-1}\left|\theta-\theta_{0}\right|^{2} d \sigma(\theta) d k .
$$

Then,

$$
\begin{aligned}
I \leq & C \int_{0}^{1} k^{d-1} \int_{S^{d-1}}\left|\widehat{\mathcal{P}_{j}(\mathbf{f})}\left(k\left(\theta-\theta_{0}\right)\right)\right|^{2} d \sigma(\theta) d k \\
& +C \int_{1}^{\infty} k^{d-1+2 \alpha} \int_{S^{d-1}}\left|\theta-\theta_{0}\right|^{2 \alpha}\left|\widehat{\mathcal{P}_{j}(\mathbf{f})}\left(k\left(\theta-\theta_{0}\right)\right)\right|^{2} d \sigma(\theta) d k \\
= & C\left(I_{1}+I_{2}\right),
\end{aligned}
$$


whenever $0 \leq \alpha \leq 1$.

In order to control $I_{1}$, we rewrite $\mathcal{P}_{j}$ in terms of the resolvent operator $R_{k}$ and the restriction operator $S_{k, \theta_{0}}$ defined in (2.23), as follows:

$$
\widehat{\mathcal{P}_{j}(\mathbf{f})}\left(k\left(\theta-\theta_{0}\right)\right)=S_{k, \theta_{0}}\left(e^{-i k \theta_{0} \cdot(\cdot)} f_{j+1} R_{k} \cdots f_{2} R_{k}\left(f_{1} e^{i k \theta_{0} \cdot(\cdot)}\right)\right)(\theta) .
$$

Using this identity we have that

$$
I_{1}=\int_{0}^{1} k^{d-1}\left\|S_{k, \theta_{0}}\left(e^{-i k \theta_{0} \cdot(\cdot)} f_{j+1} R_{k} \cdots f_{2} R_{k}\left(f_{1} e^{i k \theta_{0} \cdot(\cdot)}\right)\right)\right\|_{L^{2}\left(S^{d-1}\right)}^{2} d k .
$$

We can bound the $L^{2}\left(S^{d-1}\right)$-norm that appears in the last identity using (2.24), (2.28) and (2.20) as the following diagram illustrates

$$
L_{\delta}^{2} \ldots \stackrel{j \text {-times }}{\longrightarrow} L_{\delta}^{2} \stackrel{R_{k}}{\longrightarrow} W_{-\delta}^{2,2} \stackrel{\text { Zolesio }}{\longrightarrow} L_{\delta}^{2} \stackrel{S_{k, \theta_{0}}}{\longrightarrow} L^{2}\left(S^{d-1}\right) .
$$

Therefore, if $\delta>1$ and $\alpha$ satisfies (2.26) or (2.27) we get

$$
\begin{aligned}
I_{1} & \leq C^{2 j}\left\|f_{j+1}\right\|_{W^{\alpha, 2}}^{2}\left\|f_{j}\right\|_{W^{\alpha, 2}}^{2} \cdots\left\|f_{2}\right\|_{W^{\alpha, 2}}^{2}\left\|f_{1}\right\|_{L_{\delta}^{2}}^{2} \\
& \leq C^{2 j} \prod_{\ell=1}^{j+1}\left\|f_{\ell}\right\|_{W^{\alpha, 2}}^{2} .
\end{aligned}
$$

In order to control $I_{2}$ we need to take more advantage of oscillations, so we write $\mathcal{P}_{j}$ in terms of the operator $R_{k, \theta}$ introduced in (2.21) and the restriction operator $S_{k, \theta_{0}}$ defined in (2.23), as follows:

$$
\widehat{\mathcal{P}_{j}(\mathbf{f})}\left(k\left(\theta-\theta_{0}\right)\right)=S_{k, \theta_{0}}\left(f_{j+1} R_{k, \theta_{0}} \cdots f_{2} R_{k, \theta_{0}} f_{1}\right)(\theta) .
$$

Using this identity, and writing $w=\left|\theta-\theta_{0}\right|^{\alpha}$, we have that

$$
I_{2}=\int_{1}^{\infty} k^{d-1+2 \alpha}\left\|w S_{k, \theta_{0}}\left(f_{j+1} R_{k, \theta_{0}} \cdots f_{2} R_{k, \theta_{0}} f_{1}\right)\right\|_{L^{2}\left(S^{d-1}\right)}^{2} d k,
$$

In this case, we can bound the $L^{2}\left(S^{d-1}\right)$-norm that appears in the last identity using (2.25), (2.29) and (2.22) as the following diagram illustrates

$$
W_{\delta}^{\alpha, 2} \ldots \stackrel{(j-1) \text {-times }}{\longrightarrow} W_{\delta}^{\alpha, t_{j}} \stackrel{R_{k, \theta_{0}}}{\longrightarrow} W_{-\delta}^{\alpha, r_{j}} \stackrel{\text { Zolesio }}{\longrightarrow} W_{\delta}^{\alpha, t_{j+1}} \stackrel{S_{k, \theta_{0}}}{\longrightarrow} L^{2}\left(S^{d-1}\right),
$$

whenever there exist $r_{\ell}$ and $t_{\ell+1}$ satisfying for $\ell=1,2 \ldots j$

$$
0 \leq \frac{1}{t_{\ell+1}}-\frac{1}{2} \leq \frac{1}{d+1} \quad \text { and } \quad 0 \leq \frac{1}{2}-\frac{1}{r_{\ell}} \leq \frac{1}{d+1},
$$

$$
t_{\ell+1}<\min \left(2, r_{\ell}\right) \quad \text { and } \quad 0 \leq \frac{1}{2}+\frac{1}{r_{\ell}}-\frac{1}{t_{\ell+1}} \leq \frac{\alpha}{d} .
$$

Therefore, writing $t_{1}=2$, we have

$$
\begin{aligned}
I_{2} & \leq C^{2 j} \prod_{\ell=1}^{j+1}\left\|f_{\ell}\right\|_{W^{\alpha, 2}}^{2} \int_{1}^{\infty} k^{(d-1)\left(\frac{1}{t_{j+1}}-\frac{1}{2}\right)+\sum_{\ell=1}^{j}\left(-2+(d-1)\left(\frac{1}{t_{\ell}}-\frac{1}{r_{\ell}}\right)\right)} d k \\
& =C^{2 j} \prod_{\ell=1}^{j+1}\left\|f_{\ell}\right\|_{W^{\alpha, 2}}^{2} \int_{1}^{\infty} \frac{d k}{k^{(d-1) \sum_{\ell=1}^{j}\left(\frac{1}{r_{\ell}}-\frac{1}{t_{\ell+1}}\right)+2 j}} .
\end{aligned}
$$


Since $t_{\ell+1}$ and $r_{\ell}$ have to satisfy (2.35), the best choice to get convergence of the previous integral is

$$
\frac{1}{r_{\ell}}-\frac{1}{t_{\ell+1}}=\frac{\alpha}{d}-\frac{1}{2} .
$$

With this choice we get

$$
\begin{aligned}
I_{2} & \leq C^{2 j} \prod_{\ell=1}^{j+1}\left\|f_{\ell}\right\|_{W^{\alpha, 2}}^{2} \int_{1}^{\infty} \frac{d k}{k^{(d-1)\left(\frac{\alpha}{d}-\frac{1}{2}\right) j+2 j}} \\
& \leq C^{2 j} \prod_{\ell=1}^{j+1}\left\|f_{\ell}\right\|_{W^{\alpha, 2}}^{2},
\end{aligned}
$$

for any $j \in \mathbb{N}$ fixed, whenever

$$
\alpha>d\left(\frac{1}{2}-\frac{1}{d-1}\right),
$$

and there exist $r_{\ell}$ and $t_{\ell+1}$ satisfying (2.34), (2.35) and (2.36), for $\ell=1,2 \ldots j$. Such $r_{\ell}$ and $t_{\ell+1}$ exist if

$$
0 \leq \alpha<d / 2 .
$$

Finally, the result follows from (2.32), (2.33) and (2.37) if $\alpha \leq 1$ and satisfies (2.38), (2.39) and, (2.26) or (2.27), that is, if $\alpha$ satisfies (1.21).

In order to prove Proposition 2.2, we need the existence and uniqueness of solution of the direct scattering problem in $\in W_{-\delta}^{2,2}\left(\mathbb{R}^{d}\right)$ for any fixed wave number $k>0$. This is equivalent to prove that there exists a unique $u_{s}$, satisfying (1.6). For completeness we present here that result. The following lemma will be needed (see Theorem 6.5 in [38] or [22]).

Lemma 2.10. Let $k>0$. There exists $\delta>1$ such that

$$
\left\|R_{k} f\right\|_{W_{-\delta}^{2,2}} \leq c_{k}\|f\|_{L_{\delta}^{2}} .
$$

TheOREM 2.11. For $\alpha$ satisfying $\alpha>0$ and $\alpha>\frac{d}{2}-2$, let $q \in W^{\alpha, 2}\left(\mathbb{R}^{d}\right)$ be real valued and compactly supported. For any given $k>0$ and $\theta_{0} \in S^{d-1}$, there exists a unique solution $u_{s}=u_{s}\left(x, \theta_{0}, k\right)$ of equation (1.6) such that $u_{s} \in W_{-\delta}^{2,2}\left(\mathbb{R}^{d}\right)$ for certain $\delta>1$.

Proof. We begin by proving the uniqueness. In order to do that, we have to prove that if $u$ is a solution in $W_{-\delta}^{2,2}\left(\mathbb{R}^{d}\right)$ of $u(x)=R_{k}(q u)(x)$ with $x \in \mathbb{R}^{d}$, then $u(x)=0$ for all $x \in \mathbb{R}^{d}$.

We have that, in a weak sense

$$
\left(\Delta+k^{2}\right) u(x)=q(x) u(x), \quad x \in \mathbb{R}^{d},
$$

and $u$ satisfies the outgoing Sommerfeld condition.

Consider $M>0$ large enough such that supp $q \subset B_{M}=B(0, M)$. Multiplying (2.41) by $\bar{u}$ and integrating by parts we get

$$
-\int_{B_{M}}|\nabla u|^{2}+\int_{S_{M}} \partial_{r} u \bar{u}+k^{2} \int_{B_{M}}|u|^{2}=\int_{B_{M}} q|u|^{2},
$$

where $S_{M}=\partial B_{M}$.

From here, since $q$ is real we have that

$$
\Im \int_{S_{M}} \partial_{r} u \bar{u}=0
$$


Writing the outgoing Sommerfeld condition given in (1.2) in the following equivalent form

$$
\lim _{M \rightarrow \infty} \int_{S_{M}}\left|\partial_{r} u-i k u\right|^{2}=0
$$

and using (2.42) we get

$$
\lim _{M \rightarrow \infty} \int_{S_{M}}|u|^{2}=0
$$

From here, since $u$ is a radiating solution of equation $\left(\Delta+k^{2}\right) u=0$ in the exterior domain $\mathbb{R}^{d} / B_{M}$, we conclude using Rellich's lemma (see Lemma 2.11 in page 32 of [5]) that $u=0$ out of $B_{M}$, and by a unique continuation argument (see [16]), $u(x)=0$ for all $x \in \mathbb{R}^{d}$.

To prove existence of solution we will use Fredholm alternative. We introduce the operator $T_{k}$ defined by $T_{k} u=R_{k}(q u)$. From (2.40), since supp $q \subset B_{M}$, we have that there exists $\delta>1$ such that

$$
\left\|T_{k} u\right\|_{W_{-\delta}^{2,2}} \leq C_{k, M}\|q u\|_{L^{2}\left(B_{M}\right)} .
$$

On the other hand, from Sobolev embedding theorem we have that

$$
W^{\beta, p}\left(B_{M}\right) \underset{\text { compact }}{\hookrightarrow} L^{2}\left(B_{M}\right)
$$

whenever

$$
\beta \geq 0, \quad p>1, \quad \beta p<d, \quad 2<\frac{d p}{d-\beta p} .
$$

Using (2.43), (2.44) and (2.30), we have that $T_{k}: W_{-\delta}^{2,2} \longrightarrow W_{-\delta}^{2,2}$ is a linear compact operator whenever $\alpha>0$ and $\alpha>\frac{d}{2}-2$. Thus, Fredholm alternative ensures the existence of solution of $\left(I-T_{k}\right) u=R_{k}\left(q e^{i k \theta_{0} \cdot(\cdot)}\right)$, since we have proved that any solution of $\left(I-T_{k}\right) u=0$ in $\mathbb{R}^{d}$ satisfying the outgoing Sommerfeld radiation conditions must be the trivial solution, and $R_{k}\left(q e^{i k \theta_{0} \cdot(\cdot)}\right) \in W_{-\delta}^{2,2}\left(\mathbb{R}^{d}\right)$.

We will also need to know the behaviour of the scattered solution $u_{s}\left(x, \theta_{0}, k\right)$ when $k$ tends to zero (zero energy case). The following lemma, which can be found in [37], collects several results given in [13, 14, 15, 27].

Lemma 2.12. ([37, Proposition 2.1]) Let $q \in L^{2}\left(\mathbb{R}^{d}\right) \cap L^{r}\left(\mathbb{R}^{d}\right)$ for some $r>d / 2$, be real valued and compactly supported, and let $u_{s}\left(x, \theta_{0}, k\right)$ be the solution of (1.6). For $\delta>1$ and, we have that

1. If $d \geq 3$,

$$
\left\|u_{s}\right\|_{W_{-\delta}^{2,2}}=O\left(k^{-1}\right), \quad k \longrightarrow 0 .
$$

2. If $d=2$,

$$
\left\|u_{s}\right\|_{W_{-\delta}^{2,2}}=O\left((k \log k)^{-1}\right), \quad k \longrightarrow 0 .
$$

Proof of Proposition 2.2. Arguing as in the proof of Proposition 2.1, we split the norm to control into two pieces $I$ and $I I$, and we will just bound $I$, since $I I$ can be bounded in a similar way. In this case, making the change of variables given in (2.31), we write

$$
\begin{aligned}
I= & \int_{H_{\theta_{0}}}\langle\xi\rangle^{2 \alpha}\left|\widehat{q_{m}^{r}}(\xi)\right|^{2} d \xi \\
\leq & C \int_{0}^{\frac{1}{2}} k^{d-1} \int_{S^{d-1}} \mid \widehat{q_{m}^{r}}\left(\left.k\left(\theta-\theta_{0}\right)\right|^{2} d \sigma(\theta) d k\right. \\
& +C \int_{\frac{1}{2}}^{\infty} k^{d-1+2 \alpha} \int_{S^{d-1}}\left|\theta-\theta_{0}\right|^{2 \alpha} \mid \widehat{q_{m}^{r}}\left(\left.k\left(\theta-\theta_{0}\right)\right|^{2} d \sigma(\theta) d k\right. \\
= & C\left(I_{1}+I_{2}\right),
\end{aligned}
$$


whenever $0 \leq \alpha \leq 1$.

To estimate $I_{1}$, from the definition of $q_{m}^{r}$ given in (1.16), using Cauchy-Schwarz inequality, since $q$ is compactly supported, we get

$$
\mid \widehat{q_{m}^{r}}\left(k\left(\theta-\theta_{0}\right) \mid \leq\left\|\left(q R_{k}\right)^{m}\left(q u_{s}\right)\right\|_{L^{1}} \leq C\left\|\left(q R_{k}\right)^{m}\left(q u_{s}\right)\right\|_{L_{\delta}^{2}} .\right.
$$

We can bound the $L_{\delta}^{2}$-norm that appears in the last inequality using (2.28) and (2.20) as the following diagram illustrates

$$
W_{-\delta}^{2,2} \stackrel{\text { Zolesio }}{\longrightarrow} L_{\delta}^{2} \ldots \stackrel{(m-1) \text {-times }}{\longrightarrow} L_{\delta}^{2} \stackrel{R_{k}}{\longrightarrow} W_{-\delta}^{2,2} \stackrel{\text { Zolesio }}{\longrightarrow} L_{\delta}^{2} .
$$

Therefore, if $\delta>1$ and $\alpha$ satisfies (2.26) or (2.27) we get

$$
\left\|\left(q R_{k}\right)^{m}\left(q u_{s}\right)\right\|_{L_{\delta}^{2}} \leq C^{m}\|q\|_{W^{\alpha, 2}}^{m+1}\left\|u_{s}\right\|_{W_{-\delta}^{2,2}}
$$

thus,

$$
I_{1} \leq C^{2 m}\|q\|_{W^{\alpha, 2}}^{2(m+1)} \int_{0}^{\frac{1}{2}} k^{d-1}\left\|u_{s}\left(\cdot, \theta_{0}, k\right)\right\|_{W_{-\delta}^{2,2}}^{2} d k .
$$

Since $q \in W^{\alpha, 2}\left(\mathbb{R}^{d}\right)$, Sobolev embedding theorem guarantees that $q \in L^{2}\left(\mathbb{R}^{d}\right) \cap L^{r}\left(\mathbb{R}^{d}\right)$ with $r \in\left(\frac{d}{2}, \frac{2 d}{d-2 \alpha}\right]$ whenever $\alpha \geq 0$ and $\frac{d}{2}-2 \leq \alpha \leq \frac{d}{2}$, so we can use estimates (2.45) and (2.46) in the previous inequality to obtain

$$
I_{1} \leq C^{2 m}\|q\|_{W^{\alpha, 2}}^{2(m+1)} .
$$

On the other hand, denoting $w=\left|\theta-\theta_{0}\right|^{\alpha}$, we can write

$$
I_{2}=\int_{\frac{1}{2}}^{\infty} k^{d-1+2 \alpha}\left\|w \widehat{q_{m}^{r}}\left(k\left(\theta-\theta_{0}\right)\right)\right\|_{L^{2}\left(S^{d-1}\right)}^{2} d k .
$$

Arguing as in the proof of Proposition 2.1, to control $I_{2}$ we write $q_{m}^{r}$ in terms of the operators $R_{k, \theta}$ and $S_{k, \theta_{0}}$ introduced in (2.21) and (2.23) respectively,

$$
\widehat{q_{m}^{r}}\left(k\left(\theta-\theta_{0}\right)\right)=S_{k, \theta_{0}}\left(\left(q R_{k, \theta_{0}}\right)^{m}\left(e^{-i k \theta_{0} \cdot(\cdot)} q u_{s}\right)\right)(\theta) .
$$

From here, using (2.25), (2.29) and (2.22) as the following diagram illustrates

$$
W_{\delta}^{\alpha, t_{1}} \ldots \stackrel{(m-1) \mathrm{times}}{\longrightarrow} W_{\delta}^{\alpha, t_{m}} \stackrel{R_{k, \theta_{0}}}{\longrightarrow} W_{-\delta}^{\alpha, r_{m}} \stackrel{\text { Zolesio }}{\longrightarrow} W_{\delta}^{\alpha, t_{m+1}} \stackrel{S_{k, \theta_{0}}}{\longrightarrow} L^{2}\left(S^{d-1}\right),
$$

where $0 \leq \frac{1}{t_{1}}-\frac{1}{2} \leq \frac{1}{d+1}$ and, $r_{\ell}$ and $t_{\ell+1}$ satisfy (2.34) and (2.35) for $\ell=1,2 \ldots m$, we get

$$
\left\|w \widehat{q_{m}^{r}}\left(k\left(\theta-\theta_{0}\right)\right)\right\|_{L^{2}\left(S^{d-1}\right)}^{2} \leq C^{2 m} k^{a}\|q\|_{W^{\alpha, 2}}^{2 m}\left\|e^{-i k \theta_{0} \cdot(\cdot)} q u_{s}\right\|_{W_{\delta}^{\alpha, t_{1}}}^{2},
$$

with

$$
a=(d-1)\left(\frac{1}{t_{m+1}}-\frac{3}{2}\right)-2 \alpha+\sum_{\ell=1}^{m}\left(-2+(d-1)\left(\frac{1}{t_{\ell}}-\frac{1}{r_{\ell}}\right)\right) .
$$

We can control the norm on the right hand side of (2.50) multiplying (1.6) by $q(x) e^{-i k \theta_{0} \cdot x}$, using the operator $R_{k, \theta_{0}}$, the triangular inequality and estimate (2.29) to write

$$
\left\|e^{-i k \theta_{0} \cdot(\cdot)} q u_{s}\right\|_{W_{\delta}^{\alpha, t_{1}}} \leq C\|q\|_{W^{\alpha, 2}}\left(\left\|R_{k, \theta_{0}} q\right\|_{W_{-\delta}^{\alpha, r_{0}}}+\left\|R_{k, \theta_{0}}\left(e^{-i k \theta_{0} \cdot(\cdot)} q u_{s}\right)\right\|_{W_{-\delta}^{\alpha, r_{0}}}\right),
$$


whenever

$$
\alpha \geq 0, \quad t_{1}<\min \left(2, r_{0}\right) \quad \text { and } \quad 0 \leq \frac{1}{2}+\frac{1}{r_{0}}-\frac{1}{t_{1}} \leq \frac{\alpha}{d}
$$

Using (2.22) we get

$$
\left\|R_{k, \theta_{0}}\left(e^{-i k \theta_{0} \cdot(\cdot)} q u_{s}\right)\right\|_{W_{-\delta}^{\alpha, r_{0}}} \leq k^{b} C\left\|e^{-i k \theta_{0} \cdot(\cdot)} q u_{s}\right\|_{W_{\delta}^{\alpha, t_{1}}},
$$

with

$$
b=-1+\frac{(d-1)}{2}\left(\frac{1}{t_{1}}-\frac{1}{r_{0}}\right)
$$

whenever

$$
\alpha \geq 0, \quad 0 \leq \frac{1}{t_{1}}-\frac{1}{2} \leq \frac{1}{d+1} \quad \text { and } \quad 0 \leq \frac{1}{2}-\frac{1}{r_{0}} \leq \frac{1}{d+1},
$$

From here, using (2.6), since $b<0$, then for $k \geq 1 / 2$, we have that

$$
\begin{aligned}
\|q\|_{W^{\alpha, 2}}\left\|R_{k, \theta_{0}}\left(e^{-i k \theta_{0} \cdot(\cdot)} q u_{s}\right)\right\|_{W_{-\delta}^{\alpha, r_{0}}} & \leq \frac{A}{2^{b}} C\left\|e^{-i k \theta_{0} \cdot(\cdot)} q u_{s}\right\|_{W_{\delta}^{\alpha, t_{1}}} \\
& <\frac{1}{2}\left\|e^{-i k \theta_{0} \cdot(\cdot)} q u_{s}\right\|_{W_{\delta}^{\alpha, t_{1}}}
\end{aligned}
$$

whenever $A C \leq 2^{b-1}$.

Using (2.53) in (2.51) we obtain

$$
\left\|e^{-i k \theta_{0} \cdot(\cdot)} q u_{s}\right\|_{W_{\delta}^{\alpha, t_{1}}} \leq 2 C\|q\|_{W^{\alpha, 2}}\left\|R_{k, \theta_{0}} q\right\|_{W_{-\delta}^{\alpha, r_{0}}} .
$$

From here, since $0 \leq \frac{1}{2}-\frac{1}{r_{0}} \leq \frac{1}{d+1}(\operatorname{see}(2.52))$ we can use (2.22), to get

$$
\left\|e^{-i k \theta_{0} \cdot(\cdot)} q u_{s}\right\|_{W_{\delta}^{\alpha, t_{1}}} \leq C k^{-1+\frac{(d-1)}{2}\left(\frac{1}{2}-\frac{1}{r_{0}}\right)}\|q\|_{W^{\alpha, 2}}\|q\|_{W_{\delta}^{\alpha, 2}} .
$$

And since $q$ has compact support, we can use (2.29) to obtain

$$
\left\|e^{-i k \theta_{0} \cdot(\cdot)} q u_{s}\right\|_{W_{\delta}^{\alpha, t_{1}}} \leq C k^{-1+\frac{(d-1)}{2}\left(\frac{1}{2}-\frac{1}{r_{0}}\right)}\|q\|_{W^{\alpha, 2}}^{2} .
$$

Using this inequality and (2.50) in (2.49) we get

$$
I_{2} \leq C^{2 m}\|q\|_{W^{\alpha, 2}}^{2(m+2)} \int_{\frac{1}{2}}^{\infty} k^{-2(m+1)-(d-1) \sum_{\ell=0}^{m}\left(\frac{1}{r_{\ell}}-\frac{1}{t_{\ell+1}}\right)} d k,
$$

for any $r_{\ell}$ and $t_{\ell+1}$ satisfy (2.34) and (2.35) for $\ell=0,1 \ldots m$.

If we choose $r_{\ell}$ and $t_{\ell+1}$ satisfying (2.36), the last integral is convergent if and only if

$$
2(m+1)+(d-1)(m+1)\left(\frac{\alpha}{d}-\frac{1}{2}\right)>1,
$$

or equivalently

$$
\alpha>\frac{d}{2}-\frac{(2 m+1) d}{(m+1)(d-1)} .
$$


Therefore, for any $m \in \mathbb{N}$, we have that

$$
I_{2} \leq C^{2 m}\|q\|_{W^{\alpha, 2}}^{2(m+1)}
$$

whenever

$$
0 \leq \alpha<d / 2 \quad \text { and } \quad \alpha>\frac{d}{2}-\frac{2 d}{d-1} .
$$

Finally, the result follows from (2.47),(2.48) and (2.54) for $\alpha$ satisfying (2.5).

3. Numerical experiments. In this section we show two numerical experiments in dimension 2 that illustrate the efficiency of the proposed algorithm. We follow the discretization in [2], based on a trigonometric collocation method introduced by G. Vainikko in [43]. Note that the numerical version of the sequence (1.20) only requires the numerical approximation of some integral equations involving powers of the resolvent $R_{k}$ (see the right hand side of (1.15)-(1.16)) and the inverse Fourier transform. Both ingredients are described in [2] and therefore the adaptation to the new algorithm defined here is straightforward. We refer to [2] for implementation details.

We have considered two different examples corresponding to a piecewise constant potential (Example 1) and a smooth one (Example 2). Note that the piecewise constant potential in Example 1 does not satisfy the regularity condition in Theorem 1.1, but nevertheless the algorithm provides good results. This is somehow a numerical evidence that the regularity hypotheses are probably not sharp in Theorem 1.1.

To compute the scattering data, i.e. the far field pattern, we have implemented the numerical algorithm described in [43], with a mesh twice finer than the mesh used to solve the inverse problem. In this way we try to simulate real data to recover the potential. Given a potential $q$, we first approximate the solution $u_{s}$ of the Lippman-Schwinger equation (1.6) by trigonometric collocation, and then $u_{\infty}$ in (1.7) by quadrature. A convergence result for this numerical approximation can be found in [43] and [40].

In the experiments below we have considered a computational domain $[-2.1,2.1] \times[-2.1,2.1]$ with $N$ grid points in each variable uniformly distributed. The potential is supported in the region $[-1,1] \times[-1,1]$.

3.1. Example 1. In the first example the potential is a piecewise constant function, given by

$$
q\left(x_{1}, x_{2}\right)= \begin{cases}1.2, & \text { if }\left|x_{1}\right|+\left|x_{2}\right|<0.3 \\ 1, & \text { if } 0.7<\left|\left(x_{1}, x_{2}\right)\right|<1 \\ 0, & \text { otherwise }\end{cases}
$$

The error of the approximation is computed using the discrete $L^{2}$-norm of the difference between the numerical approximation of $q$ and the projection of the continuous functional in the mesh.

In Figure 2 we show the behaviour of the error for the discrete approximations of $q_{m, \ell}$ in log-scale when considering a mesh with $32 \times 32$ points. Each line corresponds to $q_{m, \ell}$ for fixed $m$ and represents the error ( $Y$-axis) with respect to $\ell$ ( $X$-axis). Thus, the first line (in blue) illustrates the error of $q_{1, \ell}$. We see that it decreases as $\ell$ grows and it attains the lower error for $\ell=3$.

The second line (in red) corresponds to $q_{2, \ell}$. It becomes stable for $\ell=4$. The other lines correspond to $q_{3, \ell}$ and $q_{4, \ell}$. We see that they exhibit the same behaviour as $q_{2, \ell}$. Thus, we deduce that for $m=2$ and $\ell=3$ we almost attain the minimal error.

In Figure 3 we illustrate the same as in Figure 2 but this time with a mesh grid containing $128 \times 128$ points. The behaviour is almost the same but the error becomes smaller.

In Figure 4 we show a section at $x_{2}=0$, of the graph of the potential given in Example 1 and its approximation $q_{4,7}$ considering a mesh with $N=128$.

Finally, in Figure 5 we illustrate the behaviour of the error as we consider finer meshes. To this end, we take $q_{4,7}$ as the best approximation for each mesh grid, since we have seen that larger values of $m$ and $\ell$ do not improve the error significatively. Then, we compare the error as the mesh becomes finer. This illustrates the convergence of the approximations as $N$ goes to infinity. 


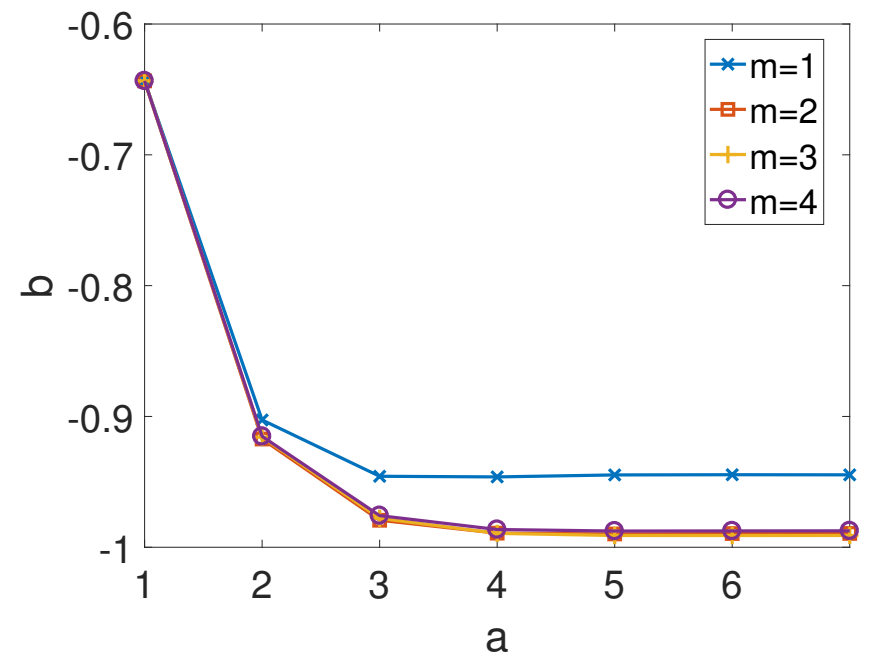

FIG. 2. Error in log-scale for Example 1 with a mesh of $32 \times 32$ points.

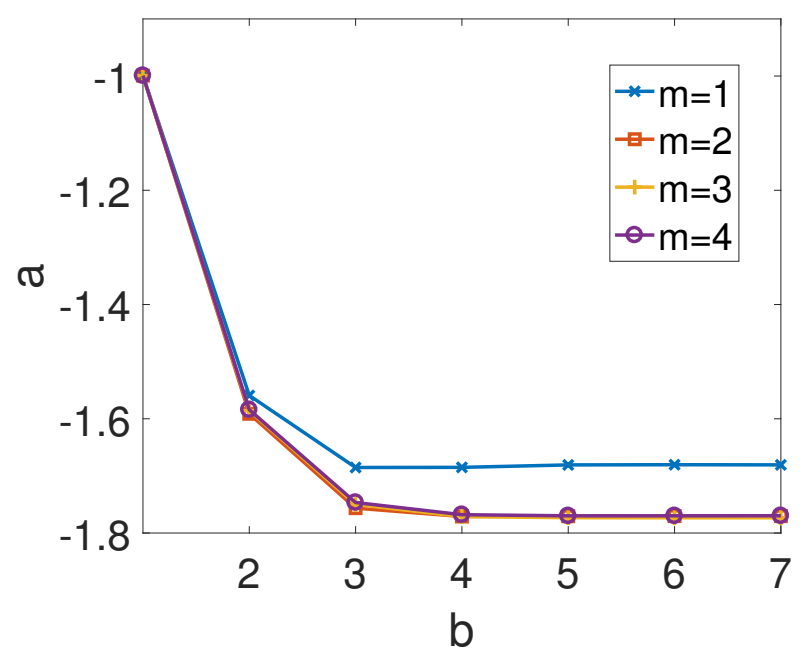

FIG. 3. Error in log-scale for Example 1 with a mesh of $128 \times 128$ points.

3.2. Example 2. Here we consider a smooth potential given by

$$
\begin{aligned}
q\left(x_{1}, x_{2}\right)= & \max \left(0, e^{-5\left|x_{1}-0.5\right|^{2}}\right)+1.5 e^{-4\left|\left(x_{1}, x_{2}\right)-(-1,0.8) / 2\right|^{2}} \\
& +2 e^{-7\left|\left(x_{1}, x_{2}\right)-0.4(-1,-1)\right|^{2}-0.4}
\end{aligned}
$$

The numerical results for this example are completely similar to those of the previous one, but the errors are significatively smaller. This is due to the regularity of the potential $q$ that we recover. We illustrate this in Figure 6, which is the analogous to Figure 5. Note that in this case we obtain errors of $10^{-4.4}$ instead of $10^{-1.8}$ of the previous example.

One of the hypothesis in Theorem 1.1 is that the size of the potential in the $W^{\alpha, 2}$ norm, with $\alpha \in(0,1)$, must be small (see Remark 1.2 above). A precise estimate of this size is not easy since it involves non-explicit 


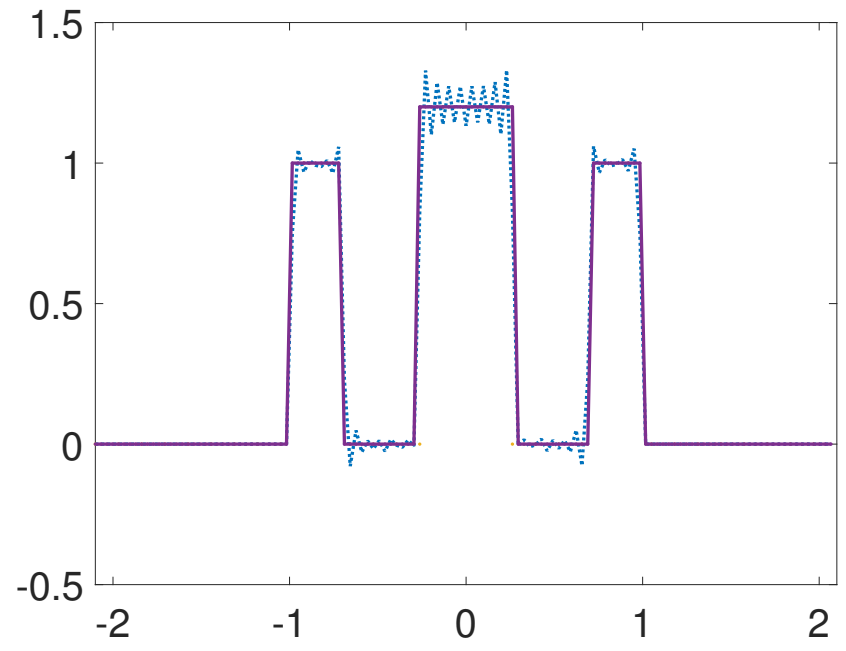

FIG. 4. Section at $x_{2}=0$ of the graph of $q$ in Example 1 and its approximation $q_{4,7}$.

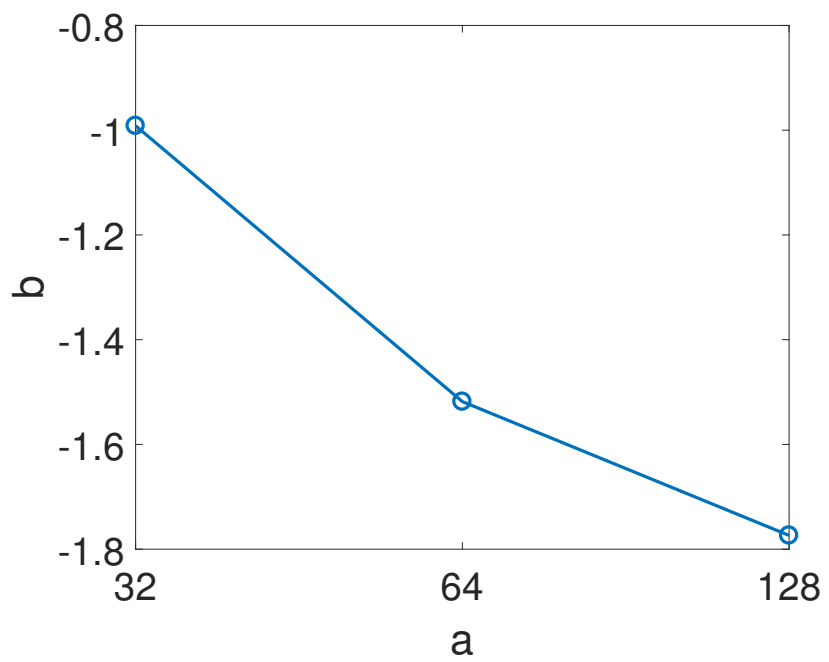

FIG. 5. Error of the approximation $q_{4,7}$ in Example 1 for different meshes.

\footnotetext{
we
}

constants that depend on certain bounds of the resolvent of the Hemholtz equation. In this second example we can give a numerical estimate of this norm for this particular potential, that we compute approximating

$$
\|q\|_{W^{\alpha, 2}}=\left(\int_{\mathbb{R}^{2}}\left(1+|\xi|^{2}\right)^{\alpha}|\widehat{q}(\xi)|^{2} d \xi\right)^{1 / 2}
$$

with the discrete Fourier Transform. We obtain,

$$
1.12 \sim\|q\|_{L^{2}}=\|q\|_{W^{0,2}} \leq\|q\|_{W^{\alpha, 2}} \leq\|q\|_{W^{1,2}} \sim 2.73, \quad 0<\alpha<1 .
$$

Therefore, the norm of the potential is not particularly small in this example. 


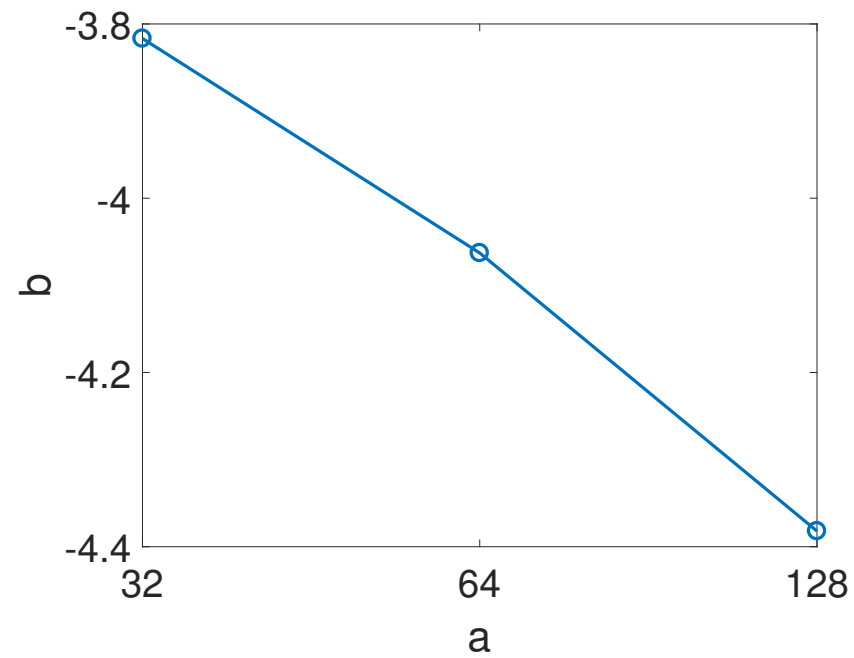

FIG. 6. Error of the approximation $q_{4,7}$ in Example 2 for different meshes.

We have tried the algorithm with potentials of the form $\lambda q(x)$ with $\lambda>1$ to understand how the algorithm works for potentials with larger norm. As $\lambda$ grows, the algorithm requires larger values of $\ell$ and $m$ to attain a stable error (as in Figure 3 for the Example 1) and the convergence rate is smaller, as $N$ grows. On the other hand, the larger value of $\lambda$ for which the algorithm converges is around $\lambda=2$.

Finally, it is worth mentioning that the numerical approximations obtained with the algorithm introduced here are similar to those obtained by the iterative one in [2], at least in the experiments that we have considered. The main point is that the computational cost is reduced drastically for large $N$ since, as we said in the introduction, we do not have to solve a Lipmann-Schwinger equation for each point in the mesh, and for each iteration.

Acknowledgments. The authors would like to gratefully thank Alberto Ruiz for his invaluable advice. We also thank Samuli Siltanen and Juan Manuel Reyes.

\section{REFERENCES}

[1] S. Agmon, Spectral properties of Schrödinger operators and scattering theory, Annali della Scuola Norm. Sup. di Pisa serie IV II (1975) 151-218.

[2] J. A. Barceló, C. Castro, J. M. Reyes, Numerical approximation of the potential in the two-dimensional inverse scattering problem, Inverse Problem 1 (2016) 015006, 19pp.

3] J. A. Barceló, D. Faraco, A. Ruiz, L. Vega, Reconstruction of singularities from full scattering data by new estimates of bilinear Fourier multipliers, Math. Ann. 346 (2010) 505-544.

[4] J. A. Barceló, A. Ruiz, L. Vega, Weighted estimates for the Helmholtz equation and some applications, Journal Funct. Anal. 150 (1997) 356-382.

[5] D. Colton, R. Kress, Inverse Acoustic and Electromagnetic Scattering Theory, Berlin (1998) Springer.

[6] G. Eskin, Lectures on linear partial differential equations, Providence (2011) Graduate Studies in Mathematics, AMS.

[7] G. Eskin, J. Ralston, The inverse backscattering problem in three dimensions, Comm. Math. Phys. 124 (1989) $169-215$.

[8] G. Eskin, J. Ralston, Inverse backscattering in two dimensions, Comm. Math. Phys. 138 (1991) 451-486.

[9] G. Eskin, J. Ralston, Inverse backscattering, J. Anal. Math. 58 (1992) 177-190.

[10] I. M. Gel'fand, B. M. Levitan, On the determination of a differential equation from its spectral function, Amer. Math. Soc. Transl. 1 (1955) 253-304.

[11] A. Greenleaf, G. Uhlmann, Recovering singularities of a potential from singularities of scattering data, Comm. Math. Phys. 157 (1993) 549-572.

[12] P. Grisvard, Elliptic problems in nonsmooth domains, Boston (1985) Pitman (Advanced Publishing Program).

[13] A. Jensen, Spectral properties of Schrödinger operators and time decay of the wave function. Results in $L^{2}\left(\mathcal{R}^{m}\right), m \geq 5$, 
Duke Math. J. 47 (1980) 57-80.

[14] A. Jensen, Spectral properties of Schrodinger operators and time decay of the wave function. Results in $L^{2}\left(\mathcal{R}^{4}\right)$, J. Math. Anal. Appl. 101 (1984) 397-422.

[15] A. Jensen, T. Kato, Spectral properties of Schrodinger operators and time decay of the wave function, Kuke Math. J. 46 (1979) 583-611.

[16] D. Jerison, C. Kening, Unique Continuation and Absence of Positive Eigenvalues for Schrodinger Operator, Annals of Math. 121(1957) 463-488.

[17] R. Jost, W. Kohn, Construction of a potential from a phase shift, Physical Rev. (2) 46 (1952) 977-992.

[18] C. Kening, A. Ruiz, C. Sogge, Uniform Sobolev inequalities and unique continuation for second order constan coefficients differential operators, Duke Math. J. (1987) 55:329-347.

[19] K. Kilgore, S. Moskow, J. C. Schotland, Inverse Born series for scalar waves, J. Comput. Math. 30 (2012) 601-614.

[20] K. Kilgore, S. Moskow, J. C. Schotland, Convergence of the Born and inverse Born series for electromagnetic scattering, Appl. Anal. 96 (2017) 1737-1748.

[21] A. Komecg, Introducction to the scattering theory for the Schrödinger equation (the Agmong-Jensen-Kato approach), Faculty of Mathematics (2009) Vienna University. available from http://www.mat.univie.ac.at/ komech/articles/sl.pdf.

[22] A. Komecg, E. Koplylova, Dispersive decay and scattering theory, Hoboken, NJ (2012) John Wiley \& Sons, Inc.

[23] K. Mochizuki, Eigenfunction expansion associated with the Schrödinger operator with a complex potential and the scattering theory, Publ. Kyoto University Series A 4 (1968)419-466.

[24] S. Moskow, J.C. Schotland, Convergence and stability of the inverse scattering series for diffuse waves, Inverse Problems 24 (2008) 065005,16.

[25] S. Moskow, J. C. Schotland, Numerical studies of the inverse Born series for diffuse waves, Inverse Problems 25 (2009) 095007, 18

[26] H. E. Moses, Calculation of the scattering potential from reflection coefficients, Physical Rev. (2) 102 (1956) $559-567$.

[27] M. Murata, Asymptotic expansionsnin time for solutions of Schrodinger-type equations, J. of Func. Analysis 49 (1982) $10-56$.

[28] R. G. Novikov, An iterative approach to non-overdetermined inverse scattering at fixed energy, Sbornik: Mathematics 206 (2015) 120-134.

[29] L. Päivärinta, V. Serov, Recovery of singularities of a multidimensional scattering potential, SIAM J. Math. Anal, 29 (1998) 697-711.

[30] L. Päivärinta, V. Serov, E. Somersalo, Reconstruction of singularities of a scattering potential in two dimension, Advances in applied mathematics, 15 (1994) 97-113.

[31] L. Päivärinta, E. Somersalo, Inversion of discontinuities for the Schrödinger equation in three dimensions, SIAM J. Math. Anal, 22 (1991) 4807-499.

[32] R. T. Prosser, Formal solutions of inverse scattering problems, J. Math. Phys. 10 (1969) 1819-1822.

[33] R. T. Prosser, Formal solutions of inverse scattering problems. II, J. Math. Phys. 17 (1975) 1775-1779.

[34] R. T. Prosser, Formal solutions of inverse scattering problems. III, J. Math. Phys. 21 (1980) 2648-2653.

[35] R. T. Prosser, Formal solutions of inverse scattering problems. IV, J. Math. Phys. 23 (1982) 2127-2130.

[36] J. M. Reyes, Problema inverso de scattering para la ecuación de Schrödinger: Reconstrucción parcial del potencial a partir de datos de retrodispersión en 2D y 3D, Tesis doctoral (2007) Universidad Autónoma de Madrid, Spain: available from http://www.uam.es/gruposinv/inversos/publicaciones/index.html.

[37] A. Ruiz, Recovery of the singularities of a potential from fixed angle scattering data, Commun. Partial Diffe. Equations 26 (2001) 1721-1738.

[38] A. Ruiz, Harmonic analysis and inverse problems. Notes of the 4th Summer School in Inverse Problems, Oulu, Finland (2002). available from http://www.uam.es/gruposinv/inversos/publicaciones/publicaciones.html.

[39] A. Ruiz, L. Vega, On local regularity of Schrödinger equations, Interna. Math. Res. Notices, 1 (1993) 13-27.

[40] J. Saranen, G. Vainikko, Periodic integral and Pseudodifferential equations with numerical approximation, Springer Monographs in Mathematics, Springer-Verlag, Berlin, 2002.

[41] P. Stefanov, Generic uniqueness for two inverse problems in potential scattering, Commun. Partial Diffe. Equations, 17 (1992) 55-68.

[42] P. A. Tomas, (1975) A restriction theorem for the Fourier transform, Bull. Amer. Math. Soc. 81 (1992) $477-478$.

[43] G. Vainikko, Fast solvers of the Lippmann-Schwinger equation, Helsinki University of Technology, Research Reports, A387 (1997) 3-18.

[44] J. L. Zolesio, Multiplication dans les espaces de Besov, Pro. Royal Soc. Edinburgh, 78A (1977) 113-117. 